\title{
Humoral immune response to islet autoantigens in Japanese patients with type 1 diabetes
}

Running Title: Anti-islet autoantibodies in Japanese type 1 diabetes

Eiji Kawasaki ${ }^{1}$, Genpei Kuriya ${ }^{2}$, Tsuyoshi Satoh $^{2}$, Keiichiro Fujishima ${ }^{2}$, Akie Moriuchi ${ }^{1}$, Keiko Fukushima $^{1}$, Masako Ozaki ${ }^{2}$, Norio Abiru ${ }^{2}$, Hironori Yamasaki ${ }^{2}$, and Katsumi Eguchi ${ }^{2}$

${ }^{1}$ Department of Metabolism/Diabetes and Clinical Nutrition, Nagasaki University Hospital of Medicine and Dentistry, Nagasaki 852-8501, Japan

${ }^{2}$ The First Department of Internal Medicine, Nagasaki University Graduate School of Biomedical Sciences, Nagasaki 852-8501, Japan

\section{Address for correspondence and reprint requests:}

Eiji Kawasaki, M.D.

Department of Metabolism/Diabetes and Clinical Nutrition,

Nagasaki University Hospital of Medicine and Dentistry

1-7-1 Sakamoto, Nagasaki 852-8501, Japan

TEL: $\quad 81-95-819-7550$

FAX: $81-95-819-7552$

E-mail: eijikawa@nagasaki-u.ac.jp 


\section{SUMMARY}

In this study, we evaluated autoantibodies to IA-2 (IA-2A), glutamic acid decarboxylase 65 (GADA) and ICA in 233 patients with type 1 diabetes $(\mathrm{M}: \mathrm{F}=90: 143$, mean duration $4.0 \pm 6.7$ yrs) as a cross sectional study. Of 233 patients with type 1 diabetes, IA-2A was detected in $58 \%$ of patients with duration within 2 weeks, $61 \%$ of patients with duration $<1 \mathrm{yr}$, $41 \%$ of patients with diabetes for $1-3 \mathrm{yrs}, 29 \%$ for $4-9 \mathrm{yrs}$, and $21 \%$ for $\geq 10 \mathrm{yrs}$. These prevalences were similar to those of ICA, while the prevalence of GADA was not influenced by duration of diabetes with positivity of $63-74 \%$. Thus, as the duration of diabetes became long, the frequency of $\mathrm{GADA}^{+} / \mathrm{IA}-2 \mathrm{~A}^{-}$patients increased and the frequency of $\mathrm{GAD}^{+} / \mathrm{IA}-2 \mathrm{~A}^{+}$patients dropped. However, the frequency of $\mathrm{GADA}^{-} / \mathrm{IA}-2 \mathrm{~A}^{+}$patients was not influenced by duration of diabetes. The prevalence of IA-2A was significantly higher in abrupt-onset group $(68 \%, \mathrm{n}=79)$ compared to slowly progressive group $(23 \%, \mathrm{n}=22)$ in new-onset patients $(\mathrm{P}=0.0001)$. However, there was no difference on the IA-2A frequency between these two groups (abrupt-onset $26 \%, \mathrm{n}=53$ vs. slow-onset $24 \%, \mathrm{n}=21$ ) in longstanding patients, suggesting that IA-2A positivity might persist in slowly progressive patients with type 1 diabetes. These results emphasize the heterogeneity of humoral autoimmunity to protein tyrosine phosphatase-like molecules, but not to GAD, in patients with type 1 diabetes. 


\section{INTRODUCTION}

Type 1A diabetes (immune-mediated) is an autoimmune disease characterized by the T-cell mediated destruction of pancreatic beta cells and the production of autoantibodies against various islet cell autoantigens ${ }^{1}$. Over the past decade, an increasing number of islet autoantigens have been identified and sequenced. Among them insulin, glutamic acid decarboxylase (GAD) and the protein tyrosine phosphatase-like molecules IA-2 are major target autoantigens to disease associated autoantibodies. At onset, IA-2 autoantibodies (IA-2A) are sometimes found in sera that are negative for $\mathrm{GADA}^{2}$, suggesting that the humoral autoimmunity is heterogenous in each case and the patterns of the changes in the levels of these autoantibodies may differ. It has been reported that ICA disappear rapidly and GADA persist after the onset of type 1 diabetes. However, there is little knowledge on the natural course of IA-2A after diagnosis. In this study, a cross sectional change of IA-2A as well as GADA and ICA were determined in Japanese patients with type 1 diabetes.

\section{MATERIALS AND METHODS}

\section{Serum Samples}

Sera were obtained from 233 Japanese patients with type 1 diabetes (90 males and 143 females). The mean age at onset and the mean duration of diabetes at the time of obtaining the sera was $21.3 \pm 15.8$ years (mean $\pm \mathrm{SD}$ ) and $4.0 \pm 6.7$ years, respectively. One hundred one patients were new-onset patients within 14 days after the onset, 31 with duration less than 1 year, 27 with duration 1-3 years, 35 with durations 4-9 years, and 39 with duration $\geq 10$ years. Patients with fulminant type 1 diabetes were excluded from this study. The diagnosis of type 1 diabetes was according to the criteria of the World Health Organization (WHO) study group ${ }^{3}$. All subjects gave informed consent, and protocols were approved by the Institutional Review Boards of the 
Nagasaki University. Sera were stored at $-20 \mathrm{C}$ until use.

\section{Autoantibody measurements}

Radioligand binding assay was used for detecting IA-2A and GADA using the cDNA for the complete cytoplasmic region of IA- 2 (amino acids 601-979) and full-length human islet GAD65, respectively, as previously described ${ }^{2,4}$."Positive" was based on the 99th percentile of sera from 204 healthy control subjects without family history of diabetes. The cut-off indices were an index of 0.018 for IA-2A and 0.028 for GADA, respectively. In the Diabetes Autoantibody Standardization Program 2005 the IA-2A and GADA assay had sensitivity of $68 \%$ and $74 \%$ and specificity of $96 \%$ and $98 \%$, respectively. ICA were detected by immunoenzymatic staining of human (blood group $\mathrm{O}$ ) pancreatic frozen sections with serial dilutions of serum samples and peroxidase-labeled protein A, as previously described ${ }^{5}$. Positive results were defined as greater than 2.5 JDF units. The lab validity, consistency, sensitivity and specificity in the 5th ICA proficiency test were $95 \%, 95 \%, 85 \%$ and $100 \%$, respectively.

\section{Statistical analysis}

Results were expressed as mean $\pm \mathrm{SD}$. Data were analyzed by the Chi-square test and Man-Whitney $U$ test. A P value less than 0.05 was considered statistically significant.

\section{RESULTS}

\section{Anti-islet autoantibodies and disease duration}

Of 233 patients with type 1 diabetes, IA-2A were detected in $58.4 \%$ of patients at onset, $61.3 \%$ of patients with duration $<1$ year, $40.7 \%$ of patients with diabetes for $1-3$ years, $28.6 \%$ for 4-9 years, and $20.5 \%$ for $\geq 10$ years, respectively (Figure 1). The prevalence for IA-2A in each 
group was similar to that of ICA. In contrast, the prevalence of GADA was not influenced by the duration of diabetes, and was detected in $72.3 \%$ of patients at onset, $74.2 \%$ of patients with duration $<1$ year, $63.0 \%$ of patients with diabetes for $1-3$ years, $74.3 \%$ for $4-9$ years, and $66.7 \%$ for $\geq 10$ years, respectively (Figure 1).

\section{Anti-islet autoantibodies and mode of disease onset}

We then evaluated the effect of mode of diabetes onset on the positivity of anti-islet autoantibodies. Patients were subclassified into two groups according to their mode of diabetes onset: an abrupt-onset group $(n=177)$, who required insulin therapy at the time of diagnosis or within 6 months after diagnosis, and a slowly-progressive group $(\mathrm{n}=56)$, who did not require insulin therapy for more than 6 months after diagnosis. Among the 177 patients with abrupt-onset, IA-2A, GADA and ICA were detected at a frequency of $54.2 \%(96 / 177), 66.1 \%(117 / 177)$ and $55.4 \%$ (98/177), respectively. In contrast, among the 56 patients with slowly-progressive form, IA-2A, GADA and ICA were detected at a frequency of $21.4 \%(12 / 56), 85.7 \%(48 / 56)$ and $41.1 \%$ (23/56), respectively. The relationship between duration of diabetes and autoantibody frequency was summarized in Table 1 . The frequency of IA-2A in abrupt-onset group at disease onset $(68.4 \%)$ was significantly higher than that in slowly-progressive patients $(22.7 \%, \mathrm{P}=0.0001)$. However, there was no difference in terms of IA-2A frequency between abrupt-onset group and slowly-progressive group in patients with duration $>4$ years $(26.4 \%$ vs. $23.8 \%)$.

\section{Combination of IA-2A and GADA}

The association between the combination of IA-2A- and GADA-positivity and disease duration was also analyzed in overall patients (Figure 2). In new-onset patients with type 1 diabetes, $46.5 \%$ of patients reacted with both GAD and IA-2, and $25.7 \%$ of patients were 
$\mathrm{GADA}^{+} / \mathrm{IA}-2 \mathrm{~A}^{-}$. Rest of patients $(\mathrm{n}=22,9.4 \%)$ were positive for IA-2A but GADA-negative at the time of autoantibody measurements. Because of the different disappearance speed of GADA and IA-2A, the frequency of GADA $/ \mathrm{IA}-2 \mathrm{~A}^{-}$patients increased and the frequency of $\mathrm{GAD}^{+} / \mathrm{IA}-2 \mathrm{~A}^{+}$ patients dropped as the duration of diabetes became long. However, the frequency of $\mathrm{GADA}^{-} / \mathrm{IA}-2 \mathrm{~A}^{+}$patients was not influenced by duration of diabetes (Figure 2).

\section{DISCUSSION}

It is well known that ICA disappear rapidly and GADA persist after the onset of type 1 diabetes, especially in type 1 diabetic patients with other organ-specific autoimmunity such as an autoimmune thyroid disease ${ }^{6}$. However, there is little knowledge on the natural course of IA-2A after diagnosis. In this study we have demonstrated that the disappearance speed of IA-2A was similar to that of ICA. However, among patients with duration $\geq 10$ years, $77.8 \%(7 / 9)$ of ICA-positive patients were negative for IA-2A, while $80.0 \%(8 / 10)$ of IA-2A-positive patients were ICA-negative, indicating the rapid decline of ICA positivity does not simply reflect the disappearance of humoral autoimmune response to IA-2. As shown in Table 1, the frequency of IA-2A in abrupt-onset group was significantly higher than that in slowly-progressive patients at onset $(\mathrm{P}=0.0001)$. This is consistent with the previous studies showing that the mode of diabetes onset is one of the factors which affect IA-2A positivity ${ }^{2,7}$. One of the interesting findings in this study was the fact that the prevalence of IA-2A in long-standing patients was similar to that in new-onset patients in slowly progressive group. Furthermore, the combined analysis of GADA and IA-2A revealed that about $10 \%$ of patients were GADA $/ / \mathrm{IA}-2 \mathrm{~A}^{+}$even after a long duration of the disease, which is similar prevalence observed in new-onset patients (Figure 2). These results lead to the speculation that humoral immune response to IA-2 might persist in slowly progress form of type 1 diabetes and GADA-IA-2A ${ }^{+}$patients. However, longitudinal analyses of IA-2A in both 
groups are required to confirm these hypotheses. In conclusion, the current findings emphasize the heterogeneity of humoral autoimmunity to protein tyrosine phosphatase-like molecules, but not to $\mathrm{GAD}$, in patients with type 1 diabetes.

\section{REFERENCES}

1. Kawasaki, E., R. G. Gill \& G. S. Eisenbarth. 1999. Type 1 diabetes. In Endocrine and Organ Specific Autoimmunity. Eisenbarth, G. S., Ed.: 149-182. R.G.Landes Co. Austin, TX.

2. Sera, Y., et al. 1999. Autoantibodies to multiple islet autoantigens in patients with abrupt onset type 1 diabetes and diabetes diagnosed with urinary glucose screening. J Autoimmun. 13: 257-265. 3. World Health Organization. 1985. Diabetes Mellitus: Report of a WHO Study Group (Tech.Rep.Ser., No.727). pp. 9-20. World Health Organization. Geneva.

4. Kawasaki, E., et al. 2003. Epitope analysis of GAD65 autoantibodies in Japanese patients with autoimmune diabetes. Ann N Y Acad Sci. 1005: 440-448.

5. Kawasaki, E., et al. 1994. Evaluation of islet-specific autoantibodies in Japanese patients with insulin-dependent diabetes mellitus: A comparison between autoantibodies to glutamic acid decarboxylase, autoantibodies to $64 \mathrm{kDa}$ islet cell protein and islet cell antibodies. J.Autoimmunity. Journal of Autoimmunity. 7: 791-802.

6. Kawasaki, E., et al. 1994. Autoantibodies to glutamic acid decarboxylase in patients with IDDM and autoimmune thyroid disease. Diabetes. 43: 80-86.

7. Yamada, K., et al. 1997. Combined measurements of GAD65 and ICA512 antibodies in acute onset and slowly progressive IDDM. Diabetes Res Clin Pract. 35: 91-98. 


\section{Figure Legends}

Figure 1 Prevalence of ICA, GADA, and IA-2A in type 1 diabetic patients with different durations of diabetes. New-onset patients represent type 1 diabetic patients with duration $<14$ days after the onset. $\quad * P<0.005, * * P<0.0001$ vs. New-onset group

Figure 2 Prevalence of corresponding type 1 diabetic patients divided by GADA and IA-2A positivity in each diabetes duration group. 
Figure 1

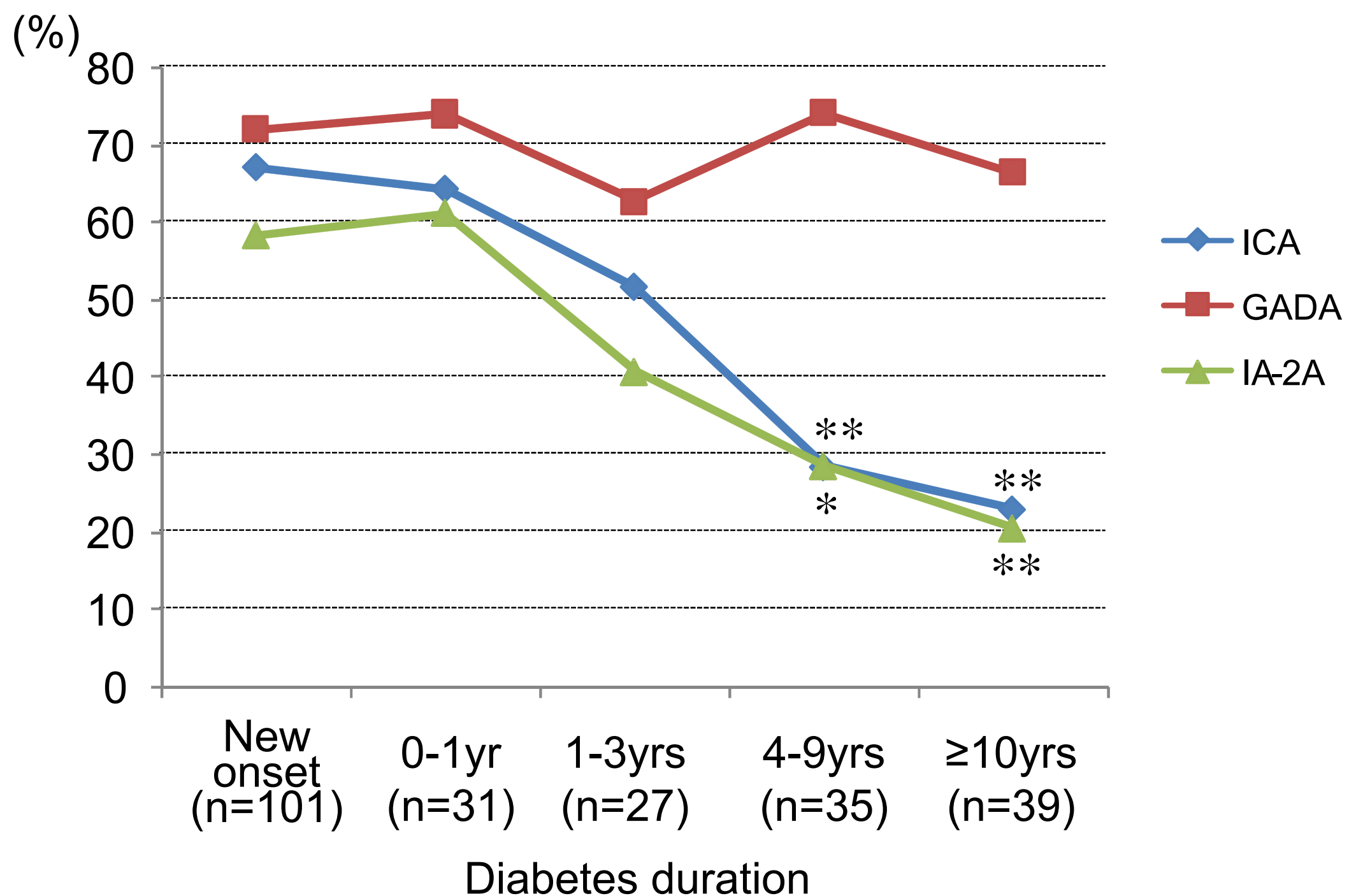

${ }^{*} \mathrm{P}<0.005,{ }^{* *} \mathrm{P}<0.0001$ vs. new-onset group 
Figure 2

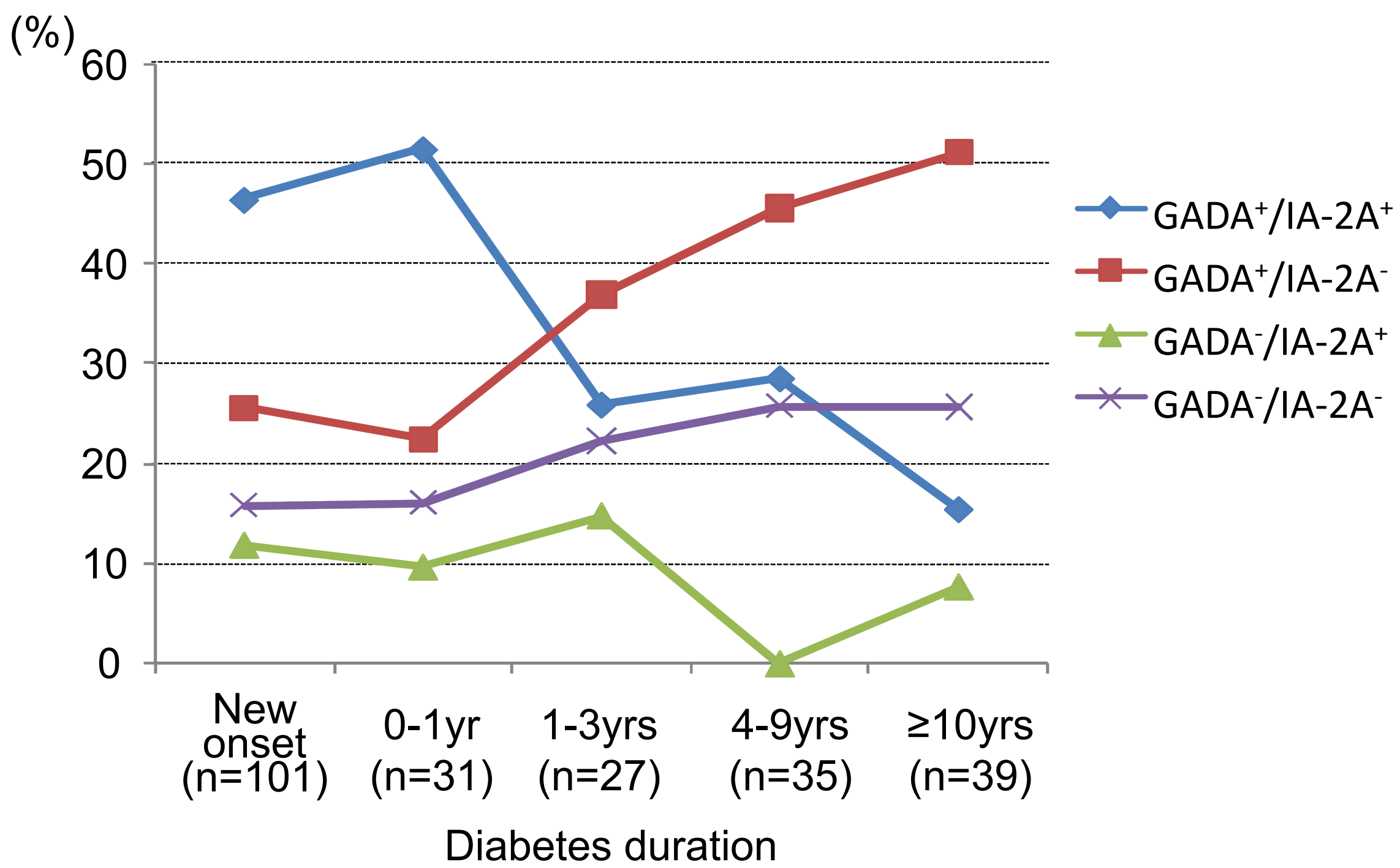


Table 1 Mode of onset and autoantibody prevalence among type 1 diabetes

\begin{tabular}{|c|c|c|c|}
\hline & New-onset & $0-3 y r s$ & $\geq 4 \mathrm{yrs}$ \\
\hline & n $(\%)$ & $\mathrm{n}(\%)$ & $\mathrm{n}(\%)$ \\
\hline \multicolumn{4}{|c|}{ Abrupt-onset $(\mathrm{n}=177)$} \\
\hline ICA & $60 / 79(75.9)^{*}$ & $27 / 45(60.0)$ & $11 / 53(20.8)^{* *}$ \\
\hline GADA & $56 / 79(70.9)$ & $29 / 45(64.4)$ & $32 / 53(60.4)$ \\
\hline IA-2A & $54 / 79(68.4)^{*}$ & $28 / 45(62.2)$ & $14 / 53(26.4)^{* *}$ \\
\hline \multicolumn{4}{|c|}{ Slowly progressive $(\mathrm{n}=56)$} \\
\hline ICA & $8 / 22(36.4)$ & $7 / 13(53.8)$ & $8 / 21(38.1)$ \\
\hline GADA & $17 / 22(77.3)$ & $11 / 13(84.6)$ & 20/21 (95.2) \\
\hline IA-2A & $5 / 22(22.7)$ & $2 / 13(15.4)$ & $5 / 21(23.8)$ \\
\hline
\end{tabular}

${ }^{*} P<0.0005$ vs. slowly progressive group; ${ }^{*} P<0.0001$ vs. new-onset patients 